\title{
La larga historia del Tesoro de las dos lenguas española y francesa de César Oudin (ed. princeps: 1607): entre autoridad e innovación
}

\section{The Long History of the Tesoro de las dos lenguas española y francesa of César Oudin (ed. princeps: 1607): Between Authority and Innovation}

\section{Marc Zuili}

Université de Versailles Saint-Quentin-en-Yvelines

(Laboratoire «DYPAC») et École Polytechnique (Palaiseau)

FRANCIA

marczuili@aol.com

[Hipogrifo, (issn: 2328-1308), 4.1, 2016, pp. 271-288]

Recibido: 29-06-2015 / Aceptado: 29-09-2015

DOI: http://dx.doi.org/10.13035/H.2016.04.01.18

Resumen. Este trabajo pretende estudiar la larga historia del Tesoro de las dos lenguas española y francesa (ocho ediciones entre 1607 y 1675) de César Oudin. Esta investigación demuestra que el hispanista francés se valió de muchos materiales lexicográficos sacados de obras anteriores que eran textos de referencia erigidos en autoridades (se trata de los diccionarios de Nebrija, de Cristóbal de las Casas, de Heinrich Hornkens y de Jean Palet). Oudin supo enriquecer estos préstamos hasta tal punto que su Tesoro... se convirtió en una obra innovadora que, a su vez, sirvió de modelo a muchos lexicógrafos posteriores como Lorenzo Franciosini, César-Joachim Trognesius, Arnaldo De la Porte, Nicolas Mez de Braidenbach y Francisco Sobrino. Por consiguiente, el Tesoro... de César Oudin es un diccionario que se sitúa claramente entre la autoridad de la tradición y el poder de la innovación.

Palabras clave. Diccionarios multilingües, siglos XVII y XVIII, César Oudin. 
Abstract. The purpose of this work is to examine the long history of the Tesoro de las dos lenguas española y francesa by César Oudin, eight editions of which were published between 1607 and 1675. Our research shows that the French hispanist used a considerable number of lexicographical sources, which were earlier authoritative works of reference. These were the dictionaries by Nebrija, Cristóbal de las Casas, Heinrich Hornkens and Jean Palet. However, Oudin enriched these sources to such a point that his Tesoro... became an innovative piece of work which, in its turn, would later become a model for a great many lexicographers such as Lorenzo Franciosini, César-Joachim Trognesius, Arnaldo De la Porte, Nicolas Mez de Braidenbach and Francisco Sobrino. This is the reason why César Oudin's dictionary can be definitly said to pertain to the authority of tradition while also relating to the power of innovation.

Keywords. Multilingual Dictionaries, 17th and 18th Centuries, César Oudin.

El Tesoro de las dos lenguas española y francesa del famoso hispanista francés César Oudin (edición princeps: París, Marc Orry, 1607) constituye, sin duda alguna, un texto pionero, ya que es uno de los primeros diccionarios bidireccionales «español-francés» y «francés-español» digno de este nombre por su gran extensión. En efecto, antes de su publicación, no circulaban más que unos léxicos bastante escuetos y la única obra que se le parecía, sin igualarlo, era el Diccionario muy copioso de la lengua española y francesa/Dictionaire (sic) très-ample de la langue françoise et espagnole de Jean Palet (edición princeps: París, M. Guillemot, 1604). En el marco de este volumen dedicado a la autoridad de la tradición y al poder de la innovación, tras establecer la lista cronológica y exhaustiva de las diferentes ediciones del Tesoro..., quisiera mostrar cómo César Oudin supo encontrar muchísimos materiales lexicográficos en varios autores anteriores cuyas publicaciones eran textos de referencia por su autoridad (Antonio de Nebrija, Cristóbal de las Casas, Heinrich Hornkens, Jean Palet). Oudin completó estos préstamos con nuevas entradas, lo que le permitió publicar en 1607 un diccionario considerado en aquel entonces una obra innovadora. Además, actualizó las sucesivas ediciones de este diccionario mediante nuevos préstamos hechos a partir de las obras de Juan Hidalgo y de Sebastián de Covarrubias. Esta labor de actualización no cesó después de la muerte de César Oudin. En efecto, su propio hijo Antoine, y, más tarde, el librero-impresor de Bruselas, Jean Mommarte, siguieron el mismo método, quitando palabras anticuadas y añadiendo nuevas entradas. Muchos lexicógrafos posteriores se valieron de este diccionario novedoso para realizar sus propias publicaciones, lo que dio lugar a verdaderos plagios: fue el caso, por ejemplo, de Francisco Sobrino, que se apoderó de una gran parte del contenido del Tesoro... de Oudin para componer el tomo I (español-francés) de su propio diccionario, publicado en 1705, el Diccionario nuevo de las lenguas española y francesa. La realización de una lista comentada de estos diccionarios que tanto deben al Tesoro... de César Oudin, convertido a su vez en texto erigido en autoridad, constituye la última parte de este trabajo. En resumidas cuentas, este estudio pretende presentar la totalidad de las ediciones del Tesoro... de César Oudin, este diccionario innovador basado en diccionarios anteriores que eran tex- 
tos de referencia, y mostrar cómo a su vez se convirtió en modelo para muchos diccionarios posteriores. Los elementos presentados aquí, que provienen en gran parte de los trabajos de varios especialistas, así como de los resultados de mis propias investigaciones ${ }^{1}$, constituyen una síntesis de la larga historia del Tesoro... de César Oudin, con sus fuentes y su «descendencia».

\section{LAS DIFERENTES EDICIONES DEL TESORO... DE CÉSAR OUDIN}

La edición princeps del Tesoro... es la siguiente:

Oudin, César, Tesoro de las dos lenguas francesa y española. Thrésor des deux langues françoise et espagnolle, Paris, Marc Orry, 1607, in-4.

La segunda edición de este diccionario dio lugar a un volumen corregido y aumentado, como lo indica el propio título:

Oudin, César, Tesoro de las dos lenguas francesa y española. Thrésor des deux langues françoise et espagnolle [...] Reveu, corrigé, augmenté, illustré \& enrichy en ceste seconde édition d'un grand nombre de dictions et phrases: \& d'un vocabulaire des mots de jargon en langue espagnolle, par le mesme autheur, Paris, Vve Marc Orry, 1616, in-4.

Esta segunda edición del Tesoro... se basa en el texto de la primera edición de la obra, pero lo corrige a veces y, sobre todo, lo completa con muchas entradas sacadas esencialmente del Tesoro de las tres lenguas francesa, italiana, y española (1609) de Girolamo Vittori -autor al que evocaré de nuevo más adelante- y del Tesoro de la lengua castellana y española (1611) de Sebastián de Covarrubias. Al final del diccionario aparece también una parte nueva titulada «Vocabulaire des mots de jargon» sacada íntegramente del Vocabulario de Germanía (1609) de Juan Hidalgo. En esta nueva edición de su diccionario, César Oudin introdujo unas 5000 entradas nuevas y enriqueció con nuevas acepciones más de 1000 palabras que ya se encontraban en la edición anterior de la obra.

La tercera edición del Tesoro... se publicó en París en 1621:

Oudin, César, Tesoro de las dos lenguas francesa y española. Thrésor des deux langues françoise et espagnolle [...]. Reveu, corrigé, augmenté, illustré \& enrichy en ceste troisiesme édition d'un grand nombre de dictions \& phrases: \& d'un vocabulaire des mots de jargon en langue espagnolle, par le mesme autheur, Paris, Adrian Tiffaine, 1621, in-4.

Esta nueva edición completa otra vez el texto de la precedente y mejora la clasificación alfabética, que, sin embargo, es menos rigurosa que la de nuestros diccionarios actuales. 
La edición siguiente, la cuarta, se publicó en Bruselas en 1625, año de la muerte de César Oudin:

OUDIN, César, Tesoro de las dos lenguas francesa y española. Thrésor des deux langues espagnole et françoise [...]. Reveu, corrigé, augmenté, illustré \& enrichy en ceste quatriesme édition [...], Bruxelles, Hubert Antoine, 1625, in-4.

El contenido de esta primera edición bruselense del diccionario de César Oudin es idéntico al de la edición parisina de 1616.

La primera edición del Tesoro... realizada después de la muerte de César Oudin por su hijo Antoine (se trataba de la quinta edición de la obra) es la siguiente:

Oudin, César, Le trésor des deux langues espagnolle et françoise de César Oudin. Augmenté sur les mémoires de son autheur. Outre un bon nombre de dictions \& de phrases: Avec une seconde partie toute nouvelle, beaucoup plus ample qu'auparavant. Le tout corrigé \& réduit en meilleur ordre, par Antoine Oudin, secrétaire interprète de sa majesté [...], Paris, Antoine de Sommaville, Augustin Courbé et Nicolas \& Jean de la Coste, 1645, in-4.

La portada de esta edición revela que Antoine Oudin pudo enriquecer de nuevo el diccionario gracias a unos documentos (llamados «mémoires») que su padre le había transmitido. Además, colocó en su debido lugar cada una de las entradas del «Dictionnaire de Xerigonça ou jargon», en vez de reagruparlas al final de la parte «español-francés» como era el caso en todas las ediciones anteriores. Además, Antoine Oudin realizó una modernización del volumen, ya que quitó varias palabras y expresiones anticuadas que habían dejado de utilizarse.

Quince años más tarde, en 1660, Juan Mommarte realizó la segunda edición bruselense del Tesoro... (se trataba de la sexta edición de este diccionario):

Oudin, César, Tesoro de las dos lenguas, española y francesa de Cæsar Oudin. Añadido conforme a las memorias del autor [...] por Antonio Oudin, secretario intérprete del Rey de Francia. Nuevamente corregido y aumentado de infinidad de omissiones, adiciones y vocablos [...], por Juan Mommarte, Bruselas, Juan Mommarte, 1660, in-4.

Jean Mommarte introdujo en este volumen varias entradas nuevas, pero muchas de ellas no son más que neologismos creados por el propio impresor o palabras específicas del español hablado en Flandes, es decir que no las utilizaban los españoles de la Penísula. En efecto, como subraya el profesor Robert A. Verdonk, «la parte "español-francés" sí contiene novedades, pero cabe advertir que en su mayor parte éstas no se corresponden con el uso de los autores [españoles] de la época»². El volumen contenía también un apéndice titulado «Vocabulario de las principales ciudades, villas, reynos, provincias y ríos del mundo». 
En este mismo año, una séptima edición del Tesoro... se publicó en Paris. Luis Pablo Núñez señala que «existen numerosas emisiones de esta edición [...] diferenciadas por su pie de imprenta» ${ }^{3}$. En efecto, se trata de una edición compartida entre varios impresores-libreros parisinos ${ }^{4}$ :

Oudin, César, Le Trésor des deux langues espagnole et françoise de César Oudin. Augmenté sur les mémoires de son auteur [...] par Antoine Oudin, secrétaire interprète de sa Majesté, Paris, Michael Bobin, 1660, in-4.

Por fin, la última edición antigua del Tesoro... vio la luz en Lyon en 1675. Dio lugar a tres emisiones publicadas simultáneamente por J.-B. Bourlier y L. Aubin, Miguel Mayer y Antoine Beaujollin:

Oudin, César, Tesoro de las dos lenguas francesa y española. Thrésor des deux langues françoise et espagnole, León de Francia, J.-B. Bourlier \& L. Aubin (o Miguel Mayer o A. Beaujollin), 1675, in-8.

La obra ya no se volvió a publicar hasta 1968, año en el que el profesor Bernard Pottier editó una versión facsímil de la parte «español-francés» del Tesoro...:

Oudin, César, Tesoro de las dos lenguas española y francesa, facsímil de la parte «español-francés» de la edición de León de Francia (1675), con una presentación de Bernard Pottier, Paris, Ediciones Hispanoamericanas, 1968.

Para ser exhaustivo, conviene señalar que dentro de poco, la editorial Honoré Champion publicará la edición íntegra del Tesoro... (con un prefacio de la profesora Dominique Reyre) en dos volúmenes, «español-francés» y «francés-español», que acabo de realizar. Se tratará de una version facsímil de la edición bruselense de 1660, precedida por un estudio muy completo sobre la historia de esta obra maestra de la lexicografía hispano-francesa y de su autor, César Oudin.

En el siglo XVII, hubo también una serie trilingüe del Tesoro... (español, francés e italiano). Este diccionario, hecho por Girolamo Vittori ${ }^{5}$ (en francés, Hiérosme Victor), conoció seis ediciones sucesivas: 1609, 1616, 1627, 1637, 1644 y 1671. Para realizar la de 1609 Vittori copió íntegramente el contenido de la edición parisina de 1607 del Tesoro... de César Oudin, introdujo muchas entradas suplementarias, y añadió la traducción italiana de todos estos vocablos. Oudin, en el «Advertissement

3. Pablo Núñez, 2010, vol. 2, p. 389.

4. Además de la emisión de Michael Bobin que cito aquí, conocemos otras once que revelan los siguientes impresores-libreros: Louis Chamhoudry, Jean Dupuis, Thomas Jolly, Simon Le Sourd, Estienne Maucroy, P. Ménard, Pierre Moet, la viuda Edme Pepingué, Pierre Rocollet, Antoine de Sommaville, Jacques Villery.

5. Annamaria Gallina nos proporciona muchos detalles sobre la vida de Vittori: «Girolamo Vittori nacque a Bologna prima del 1549 [...]. Nel 1567, a causa delle persecuzioni contro i riformati, dovette trasferirsi con la famiglia a Ginevra. Qui nel 1573 sposò Camilla Arnolfini, di nobile famiglia lucchese, dalla quale ebbe sette figli. II 30 dicembre 1574 ottenne la "bourgeoisie" di Ginevra e continuò a risiedere in quella città. Nel 1609 [...] esce il "Tesoro de las tres lenguas" che lo farà accusare di plagio dai posteri. [...] È ignoto l'anno della sua morte» (Gallina, 1959, pp. 230-231). 
nécessaire aux lecteurs» de la segunda edición de su Tesoro... (1616), denunció por plagio a Vittori, pero sin designarlo explícitamente:

I'adiousteray à l'aduertissement cy dessus, que ceux qui ont fait imprimer ce Tesoro [i.e. la edición trilingüe] à Genèue, avec l'addition de la langue italienne, \& de quelques dictions espagnoles fournies par d'autres, n'ont pas oublié de s'attribuer l'honneur de l'auoir compilé, mais ils ne se sont pas aduisez de le conférer avec d'autres dictionnaires, tant françois qu'espagnols, où il eussent trouué, qu'en la première édition faite à Paris, il manque (par la faute des imprimeurs) une page entière en la lettre $R$, de la seconde partie qui est françoise-espagnole, à sçauoir depuis le mot retordre iusques à rêuer, preuue suffisante, puisque le mesme deffault est en leur impression, qu'ils ne se sont seruis que de notre exemplaire ${ }^{6}$.

Y César Oudin completó su ataque contra Vittori con las palabras siguientes:

En outre, i'eusse désiré que celuy qui y a adiousté l'italien, eut mieux entendu les deux autres langues, car i'ay trouué qu'en l'explication seule de ce qui n'est pas mesme une lettre entière, il a laissé eschaper de sa plume cinq ou six absurditez?

Volviendo al Tesoro... de César Oudin, la lista de sus diferentes ediciones es reveladora, por su extensión, del enorme éxito de este diccionario a lo largo del siglo XVII. Publicado con actualizaciones durante casi setenta años, constituyó una obra innovadora y una obra maestra de la lexicografía bilingüe franco-española de la época, que sirvió a su vez de modelo para las publicaciones posteriores de varios lexicógrafos. Su mayor mérito, que le permitió perdurar tanto, es que supo evolucionar continuamente. En efecto, tal como vamos a ver, César Oudin realizó la primera edición del Tesoro... gracias a los materiales que figuraban en diccionarios anteriores que eran ya obras de referencia, es decir, con autoridad. Pero el lexicógrafo francés introdujo nuevos vocablos que sacó de diversos textos literarios españoles que había leído, lo que convirtió su diccionario en una obra innovadora. Por ejemplo, a propósito de la palabra «follón», después de dar los equivalentes «Un faineant, un batteur de pavé \& lâche poltron [...]» , proporcionaba a sus lectores la indicación siguiente:

le l'ay trouvé en Don Quixote en la signification que j'ay mise ci-dessous, \& ainsi que les traduisent tous les romans françois, ausquels j'ay souvent trouvé ces mots, félon \& ord pautonnier, en parlant à des géants, qui sont ordinairement introduits pour hommes félon, cruels et vilains 9 . 
De la misma manera, para el vocablo «alinde», precisa a sus lectores «le l'ay trouvé dedans les œuvres de Boscán» ${ }^{10}$. Además, como ya hemos visto, Oudin, muy al tanto de lo que se publicaba en España, también sacó partido de dos obras capitales, para extraer de ellas nuevas entradas que introdujo en la segunda edición de su Tesoro...: el Tesoro de la lengua castellana y española (1611) de Sebastián de Covarrubias y el Vocabulario de Germanía (1609) de Juan Hidalgo. Se apropió también del vocabulario complementario que Vittori había introducido en su diccionario trilingüe y lo incluyó en la edición de 1616 de su Tesoro de las dos lenguas española y francesa, lo que significa que César Oudin, plagió a su propio plagiario, como quedó demostrado por el especialista en lexicografía, Louis Cooper ${ }^{11}$. Tras la muerte de César Oudin, su hijo Antoine, y, más tarde, el librero-impresor bruselense Jean Mommarte, enmendaron y enriquecieron el Tesoro... con el propósito de mantenerlo actualizado y vigente.

Para comprender mejor la evolución del Tesoro... de César Oudin, vamos a estudiar detalladamente las fuentes que utilizó el lexicógrafo francés: estos diccionarios anteriores, que formaban parte de una larga y rica tradición lexicográfica, constituyeron los modelos autoritativos de los que se valió Oudin.

\section{EL TESORO... DE CÉSAR OUDIN: UNA OBRA LEXICOGRÁFICA INNOVADORA BASADA EN TEXTOS ANTERIORES ERIGIDOS EN AUTORIDADES}

El «Advertissement nécessaire aux lecteurs» de la segunda edición del Tesoro... (1616) contiene la única alusión de César Oudin a unos lexicógrafos anteriores:

le diray aussi un mot quant à l'ordre que j'ay tenu (suiuant celuy qu'ont obserué le Nebricense, Cristóual de las Casas, \& Couarruuias aussi) en la disposition des lettres... ${ }^{12}$

Esta lacónica frase revela que Oudin conocía muy bien los trabajos de estos famosos lexicógrafos, pero no indica hasta qué punto los había explotado para realizar y, más tarde, enriquecer las diferentes ediciones de su propio diccionario bilingüe.

En tal sentido, hay que agradecer a dos especialistas en lexicografía, Louis Cooper y Robert A. Verdonk, el habernos proporcionado una serie de informaciones de gran importancia acerca de los diccionarios de los que se valió César Oudin para realizar su Tesoro....13. Gracias a estos dos investigadores sabemos ahora que el lexicógrafo francés extrajo gran parte de los vocablos que figuran en la primera edición de su diccionario bilingüe del Diccionario muy copioso de la lengua española y

\footnotetext{
10. Oudin, Tesoro..., 1675, p. 52.

11. Cooper, 1960a.

12. Oudin, Tesoro..., 1616, fols. ã III v - ã IV.

13. Cooper, 1960a, 1960b, 1962a, 1962b; Verdonk, 1990, 1992.
} 
francesa/Dictionaire très-ample de la langue françoise et espagnole de Jean Palet ${ }^{14}$ (ed. princeps: Paris, Matthieu Guillemet, 1604):

Salvo algunas pequeñas diferencias en las definiciones (leves cambios estilísticos o de significado), y con excepciones de ciertas omisiones en 01 [= parte «español-francés» del Tesoro... de Oudin] de artículos de P1 [= parte «españolfrancés» del Diccionario muy copioso... de Palet] (en especial palabras terminadas en -or [...]) o de algunas explicaciones que Oudin consideró erróneas o insuficientes, el texte de 01 contiene prácticamente todo lo aprovechable de P1. En la mayoría de los casos, los dos textos son idénticos (o casi idénticos, pues Oudin amplía cierto número de glosas) ${ }^{15}$.

Como la obra de Palet ya había retomado una gran parte del contenido del Recueil de dictionnaires françoys, espaignolz et latins, obra de Heinrich Hornkens ${ }^{16}$ publicada en Bruselas en 1599 por el librero R. Velpius, es evidente que este Recueil... constituye también una fuente indiscutible del Tesoro... de César Oudin. Pero éste no se conformó con utilizar los materiales contenidos en el diccionario de Palet, sino que recurrió directamente a la fuente misma de esta obra, el Recueil... de Hornkens, para recoger unos vocablos más, olvidados o eliminados por Palet:

El Recueil de Hornkens fue la fuente de la mayor parte del Diccionario muy copioso de la lengua española y francesa [...] de Joan Palet, y también la fuente

14. Jean Palet era médico ordinario de Henri de Bourbon, príncipe de Condé. En los preliminares de su Diccionario muy copioso... nos revela que, por culpa de una grave herida, estuvo en cama durante tres años durante los cuales realizó este diccionario: «Este trauajo, Señor mio, es la cuenta que yo doy a V. Exc. de tres años que mi fastidiosa herida me ha tenido continuamente enclauado en una cama como ynutil, y que ha ensalmado parte de mis dolores con la dulzura de tan agradable diuertimiento» (Dedicatoria al príncipe, fol. a VI). En origen, este diccionario se destinaba únicamente al uso personal de su dueño: «[...] yo no emprendi aquesta tarea sino por obedecer a los que sobre mi tienen todo el poder [...] sin algun desinio de darla a la emprenta» (Prefacio al lector, fol. a VIII v). Pero Palet indica: «Despues yo he sido como forzado a mudar de parecer por las honrradas amonestaciones de Joan de Herrera Gentilhombre Español, el qual no solo me ha estimulado a hacerlo, mas tan liueralmente me ha comunicado su assitencia que me ha ayudado a llegarla al punto en que la veis, corrigiendo mis faltas, y haciendo añadir muy mucho, como aquel que es mas docto en su lengua que otro qualquiera que hallarse pueda» (Prefacio al lector, fol. a VIII v).

15. Cooper, 1962a, pp. 317-318.

16. Debemos a uno de los artículos del profesor Verdonk unos datos muy importantes sobre Heinrich Hornkens y sobre los motivos que le condujeron a redactar su Recueil: «H. Hornkens n'est pas un Espagnol, mais un "Flamenco", originaire de Bois-le-Duc (ville qui à l'époque faisait partie des Pays-Bas espagnols), qui a appris la langue espagnole en Espagne durant un séjour de plus de dix ans à la cour de Philippe II. Lorsqu'en 1596 I'Archiduc Albert, qui réside également à cette cour, est nommé gouverneur général des Pays-Bas, Hornkens fait partie de la suite qui l'accompagne à Bruxelles. Peu de temps avant sa mort, Philippe II décide de donner sa fille Isabelle en mariage à Albert et d'octroyer à ceux-ci une très large autonomie quant au gouvernement des Pays-Bas espagnols. Bruxelles voit ainsi surgir en son sein une véritable cour avec des souverains hispanophones. Les membres de l'aristocratie "belge", de même que les nombreux généraux et officiers étrangers, qui font partie de l'Ejército de Flandes, fréquentent cette cour et veulent apprendre la langue espagnole. C'est pour répondre à leur demande que Hornkens rédige son dictionnaire» (Verdonk, 1991, p. 2977). 
principal del Tesoro de las dos lenguas española y francesa [...] de César Oudin. 0 dicho más exactamente: Palet plagió a Hornkens y fue plagiado a su vez por Oudin, quien además se sirvió directamente del diccionario de Hornkens ${ }^{17}$.

Si consideramos que el Recueil... de Hornkens se había inspirado ampliamente en los famosos diccionarios de Nebrija ${ }^{18}$ y de Cristóbal De las Casas ${ }^{19}$, podemos afirmar que el Tesoro... de César Oudin, de una manera indirecta esta vez, también se apoya en estos dos diccionarios que constituían a su vez verdaderas autoridades inscritas en una larga tradición lexicográfica. Fundado en estas obras prestigiosas, el Tesoro... de César Oudin constituye, como subraya Louis Cooper, «el mayor esfuerzo realizado hasta entonces en el terreno de la lexicografía española» ${ }^{20}$

Como acabamos de ver, César Oudin, sin confesarlo, había utilizado para la primera edición de su Tesoro... (1607) varios trabajos lexicográficos anteriores que eran ya textos de referencia. El estudio de la segunda edición de este diccionario (París, 1616) revela la presencia de 5000 entradas nuevas, así como la introducción de precisiones y nuevas traducciones para muchos términos. ¿De dónde provenían estas ampliaciones y mejoras? Una vez más debemos a Louis Cooper la respuesta a esta pregunta. En efecto, en uno de sus artículos ${ }^{21}$, este investigador establece que más de 900 nuevas entradas de la segunda edición del Tesoro... (1616) provienen del Tesoro de la lengua castellana o española de Sebastián de Covarrubias, diccionario monolingüe publicado por primera vez en 1611. En otro estudio ${ }^{22}$, Cooper revela otra fuente, muy sorprendente, de estas adiciones. Recordemos las airadas quejas de César Oudin contra Girolamo Vittori. Si bien es verdad que, como ha quedado señalado, Vittori había plagiado la primera edición del Tesoro... de César Oudin para realizar su diccionario trilingüe, no es menos cierto que introdujo en esta obra más de 3000 entradas nuevas que no figuraban en el diccionario del lexicógrafo francés. Louis Cooper confiesa su asombro frente a «estas adiciones,

17. Cooper, 1962a, p. 299.

18. No es necesario presentar a Antonio de Nebrija (1444-1522), que participó en la publicación de la Biblia políglota complutense y fue también el autor de diversas obras (filología, gramática, teología, etc.). Entre estas obras destacan un diccionario «latín-español», el Lexicon ex sermone Latino in Hispaniensem (Salamanca, 1492) y un diccionario «español-latín», el Vocabulario de romance en latín, también llamado Dictionarium ex Hispaniensi in Latium sermonem (Salamanca, 1495). Esta obra lexicográfica «si dimostrò subito tanto superiore alle sue contemporanee e più antiche consorelle da essere presa come fondamento o modello da gran parte dei vocabolari posteriori bilingui o plurilingui, anche di autore straniero» (Gallina, 1959, p. 13).

19. Cristóbal De Las Casas es el autor de un Vocabulario de las dos lenguas toscana y castellana (ed. princeps: Sevilla, Francisco de Aguilar, 1570) cuyo modelo era el Vocabulario de romance en latín de Nebrija. La obra de Las Casas cuenta con casi 15000 entradas en la parte «italiano-español» y unas 10000 entradas en la parte «español-italiano». El éxito de esta obra fue tan importante que conoció muchísimas otras ediciones realizadas entre 1576 y 1620. Los datos sobre el autor de este Vocabulario escasean: oriundo de Sevilla, poeta, filólogo y lexicógrafo, fue secretario del duque de Alcalá y viajó por Italia. Murió en Sevilla en 1576 o en 1583, durante una epidemia de peste.

20. Cooper, 1960b, p. 365.

21. Cooper, 1960b.

22. Cooper, 1960 a. 
ya que Vittori nada dice de ellas ni se vanagloria de tal labor original» ${ }^{23}$. Pero lo más curioso es que Cooper revela que, a su vez, «Oudin había aprovechado casi las tres cuartas partes de estas adiciones, en la segunda edición de su Tesoro» ${ }^{24}$. Este investigador concluye su estudio con la descripción de este proceso de plagio mutuo «Oudin plagió a Vittori tan decididamente come éste le había plagiado antes a él, y sin remordimiento alguno, justificando su acción como natural represalia» ${ }^{25}$.

Por fin, señalemos que Oudin se apropió de la totalidad del Vocabulario de Germanía (1609) de Juan Hidalgo, una lista de vocablos españoles específicos de la lengua del hampa, para integrarla, con sus equivalentes en francés, en la edición de 1616 de su Tesoro...

Los diferentes elementos que acabo de presentar conducen a la siguiente conclusión: el Tesoro... de César Oudin es una obra procedente de una larga tradición de diccionarios y el lexicógrafo francés supo extraer, con mucha habilidad, gran número de entradas que estas obras autoritativas contenían. Por consiguiente, el Tesoro... se realizó mediante la yuxtaposición más o menos visible de estos materiales que ya figuraban de forma idéntica o muy similar en varias obras anteriores: el Vocabulario de romance en latín, también llamado Dictionarium ex Hispaniensi in Latium sermonem de Nebrija (ed. princeps: Salamanca, 1495), el Vocabulario de las dos lenguas toscana y castellana de Cristóbal de las Casas (ed. princeps: Sevilla, Francisco de Aguilar, 1570), el Recueil de dictionnaires françoys, espaignolz et latins de Heinrich Hornkens (ed. princeps: Brussels, Rutgerius Velpius, 1599), el Diccionario muy copioso de la lengua española y francesa de Jean Palet (ed. princeps: Paris, Matthieu Guillemot, 1604), el Tesoro de la lengua castellana o española de Sebastián de Covarrubias (ed. princeps: Madrid, Luis Sánchez, 1611), el Vocabulario de Germanía de Juan Hidalgo (ed. princeps: Barcelona, 1609) e incluso el Tesoro de las tres lenguas francesa, italiana y española de Girolamo Vittori (ed. princeps: Genève, P. Albert \& A. Pernet, 1609).

Si, como acabamos de ver, César Oudin había encontrado la mayor parte de los vocablos de su Tesoro... en varios diccionarios que constituían obras de referencia, es decir textos erigidos en autoridades, este diccionario bilingüe se convirtió muy rápidamente en un modelo innovador que, a su vez, sirvió de fuente a muchos lexicógrafos posteriores. Estos se aprovecharon del contenido del Tesoro... de Oudin para dar lugar a una larga «descendencia» de diccionarios.

\section{EL TESORO... DE CÉSAR OUDIN: DE TEXTO INNOVADOR A OBRA CON AUTORIDAD}

El éxito que conoció el Tesoro... de César Oudin se puede constatar no solamente por sus múltiples ediciones a lo largo del siglo XVII, sino también por el hecho de que, como afirma el profesor Verdonk, «de nombreux lexicographes bilingues ou plurilin-

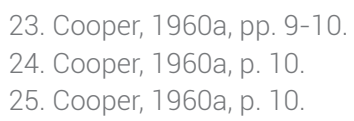


gues l'ont pris comme source ou l'ont tout simplement plagié»» ${ }^{26}$. Samuel Gili Gaya, en la «Introducción» de su Tesoro lexicográfico, va más allá cuando escribe que

Su influencia fue tan grande que puede decirse que todos los diccionarios bilingües deben algo al Thrésor de Oudin, y algunos se limitan a copiarle o traducirle con pocas alteraciones ${ }^{27}$.

Y, efectivamente, ya hemos visto cómo Girolamo Vittori se había aprovechado de la primera edición del Tesoro... para realizar su propio diccionario trilingüe. Pero no fue el único en plagiar la obra del lexicógrafo francés: entre los que se inspiraron en mayor o menor medida del Tesoro... de Oudin figuran el italiano Lorenzo Franciosini, el impresor de Amberes César-Joachim Trognesius, el flamenco Arnaldo De la Porte así como el austriaco Nicolas Mez de Braidenbach y el español Francisco Sobrino. El análisis de los diccionarios realizados por dichos autores permite ver hasta qué punto el Tesoro... de César Oudin, que hasta entonces se consideraba un texto en gran parte innovador, se había convertido ya en modelo, es decir en texto con autoridad. El análisis de todos estos diccionarios permite poner de relieve su deuda con la obra pionera de Oudin.

Lorenzo Franciosini es el autor de numerosos libros, entre los que destaca uno de los más famosos diccionarios bilingües «italiano-español» y «español-italiano» de los siglos XVII y XVIII. La edición princeps de esta obra se compone de un primer volumen titulado Vocabolario italiano e spagnolo [...] con tutte le voci toscane in castigliano e le castigliane in toscano... (668 páginas) y un segundo volumen, el Vocabulario español e italiano, aora nuevamente sacado a luz... (784 páginas). El impresor romano Giovanni Paolo Profilio publicó por primera vez esta obra en 1620. Un estudio realizado en 1959 por Annamaria Gallina demuestra que las fuentes utilizadas por Franciosini son múltiples, pero que el Tesoro... de César Oudin ocupa un puesto muy importante entre ellas, ya que muchos vocablos que aparecen en el Vocabulario español e italiano... están sacados directamente del diccionario del lexicógrafo francés:

È evidente che il Franciosini non si contentò di consultare il Las Casas, ma si rivolse anche a vocabolari spagnuoli d'altro genere e, primo di tutti, al Nebrija. [...] Anche dal Covarrubias il Franciosini prese voci e locuzioni. [...] Ė i vocaboli che non sono stati tolti dal Covarrubias, possiamo trovarli in altri dizionari, soprattutto nell'Oudin ${ }^{28}$.

Si bien es cierto que Lorenzo Franciosini se inspiró en varias fuentes, y más particularmente en el Tesoro... de Oudin, no obstante parece justo reconocer que este lexicógafo italiano realizó, al fin y al cabo, una obra bastante personal: en efecto, a pesar de que su diccionario bebía de un conjunto de obras anteriores, supo con gran habilidad distribuir de otra manera, aumentar y enriquecer estos materiales para llegar a publicar un diccionario original y muy completo que conoció muchas ediciones sucesivas durante los siglos XVII y XVIII (1620, 1636, 1637, 1638, 1645, $1665,1666,1706,1707,1735,1743,1774$ y 1796). 
El impresor C.-J. Trognesius publicó en Amberes entre 1639 y 1646 varias ediciones de un Grande dictionario y thesoro de las tres lenguas española, francesa y flamenca, que debe mucho al Tesoro... de César Oudin. Esta obra en tres tomos, también conocida bajo el título Anónimo de Amberes, dio lugar a un estudio analítico de Robert A. Verdonk en el que demuestra que gran parte de esta obra proviene directamente del Tesoro... de César Oudin ${ }^{29}$. Según este especialista, dos de los tres volumenes del Anónimo de Amberes están sacados de la edición bruselense de 1625 de la obra de Oudin:

(1) La partie Espagnol-Français-Néerlandais [...] : toutes les entrées espagnoles et leur équivalent français sont un plagiat presque littéral de la 4e édition (Bruxelles, Mommart, 1625) de C. Oudin. Quant aux équivalents néerlandais qui y ont été ajoutés (de manière très littérale d'ailleurs), ils sont l'œuvre, soit de l'imprimeur Trognesius lui-même, soit d'un de ses collaborateurs. (2) La partie Français-Espagnol [...] est encore moins originale. II s'agit à nouveau d'un plagiat de la 4e édition de C. Oudin, cette fois-ci dans sa partie F[rançais]-E[spagnol]. Trognesius a copié systématiquement toutes les entrées françaises de Oudin, sauf les sept premières, probablement pour donner l'impression qu'il s'agissait d'un dictionnaire différent. Les équivalents espagnols sont eux aussi plagiés de Oudin. Les seules différences consistent en quelques modifications de type graphique et des suppressions de I'un ou l'autre équivalent espagnol, lorsque Oudin en donne plusieurs ${ }^{30}$.

Tal análisis revela la importancia del plagio que sufrió en esta ocasión la obra de César Oudin. Pero las cosas van aún más allá. En efecto, en 1659, Arnaldo De la Porte publicó en Amberes un Nuevo dictionario o thesoro de la lengua española y flamenca, que no era más que una copia exacta del Anónimo de Amberes: este diccionario constituye, de manera indirecta esta vez, un nuevo plagio del Tesoro... de César Oudin y, por consiguiente, como subraya el profesor Verdonk, «la parte "español-neerlandés" de De la Porte no ofrece, por tanto, ningún léxico español nuevo respecto a Oudin» ${ }^{31}$.

En 1670, Nicolas Mez de Braidenbach publicó el primer diccionario bilingüe «español-alemán», el Diccionario muy copioso de la lengua española y alemana... (Viena de Austria, Juan Diego Kürner, 1670). En primer lugar, tenemos que resaltar la honestidad del autor al reconocer en la portada de su diccionario que este volumen está «sacado de diferentes autores con mucho trabajo y diligencia». Pero esta aparente honradez es bastante relativa ya que no aparece ninguna aclaraci ón acerca de estos autores. Dos especialistas han estudiado el contenido de esta obra. El primero, Franz Josef Hausmann, opina que el diccionario compuesto en Viena es una adaptación muy fiel de la edición parisina de 1607 del Tesoro de las dos lenguas española y francesa de César Oudin ${ }^{32}$. En cambio, el segundo, Dieter Messner, aunque reconoce que es posible que el diccionario de Mez de Braidenbach derive

\section{Verdonk, 1988.}

30. Verdonk, 1991, pp. 2978-2979.

31. Verdonk, 1992b, p. 35.

32. «Offenbar hat unserem Verfasser nicht die von Antoine Oudin überarbeitete Fassung von 1645 [...] vorgelegen, sondern die alte von 1607» (Hausmann, 1984, p. 170). 
del Tesoro... de Oudin - «constatamos que Oudin pudo ser el modelo» $»^{33}$, escribe-, añade que también se parece mucho al Diccionario muy copioso de la lengua española y francesa de Jean Palet por su título, por el grafismo de su portada y, sobre todo, por las entradas muy similares que se encuentran en los dos diccionarios. El profesor Messner concluye escribiendo: «creo que Palet 1604 (o Palet 1606) ha servido también de modelo a Mez de Braidenbach 1670, y no sólo Oudin 1607 como afirma Hausmann» ${ }^{34}$. Me parece que es posible conciliar la posición de cada uno de estos dos especialistas si tenemos en cuenta que el Tesoro... de César Oudin no es más que un calco algo revisado, corregido y aumentado del Diccionario muy copioso... de Jean Palet...

El último lexicógrafo evocado aquí es Francisco Sobrino, autor del Diccionario nuevo de las lenguas española y francesa, obra publicada por primera vez en 1705 y que dio lugar a un total de seis ediciones en el transcurso del siglo XVIII. Fue un diccionario tan popular y tan difundido en aquella época que Daniel M. Sáez Rivera afirma:

Popularmente, ya se debía de conocer el diccionario de Sobrino directamente como «el Sobrino», al igual que en la actualidad el Diccionario del español actual de Seco et alii es conocido como «el Seco» o el Diccionario crítico etimológico castellano e hispánico de Corominas y Pascual sea conocido en el mundillo filológico directamente como «el Corominas» ${ }^{35}$.

En cambio, según escribe Daniel M. Sáez Rivera, «muy poco es lo que sabemos sobre Francisco Sobrino» ${ }^{36}$. Sea como fuere, Francisco Sobrino, que se presentaba como oficial del Ejército de Flandes y maestro de lengua española en la corte de Bruselas, era un autor conocido por sus numerosos plagios:

Cada vez que Amado Alonso lo cita [...] es para denunciar su apropiación de la gramática de español para franceses de Oudin en la Nouvelle grammaire espagnole (1697), que publicó Sobrino con gran éxito. Aquilino Sánchez tambien subraya su carácter de plagiador [...]: así, señala que Sobrino no sólo copia de Oudin su Nouvelle grammaire espagnole (1697), sino también el Diccionario nuevo de las lenguas española y francesa (1705) y sus Diálogos nuevos en español y francés (1708). [...] Alvar Ezquerra [...] recuerda que además del diccionario y la gramática, Sobrino también copió las nomenclaturas de César Oudin y de su hijo Antoine ${ }^{37}$.

Tal unanimidad por parte de estos especialistas es reveladora de los numerosísimos préstamos tomados por Francisco Sobrino de la obra de Oudin. Y de hecho Daniel M. Sáez Rivera ha calculado que Oudin aporta el 70\% del tomo I [españolfrancés] del Diccionario nuevo de las lenguas española y francesa ${ }^{38}$. Sin embargo, el profesor Verdonk adopta una posición menos crítica hacia Sobrino. En efecto, si

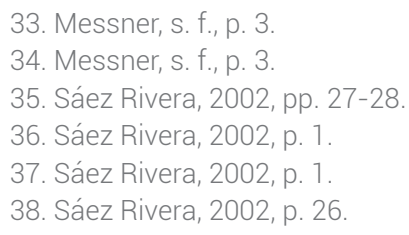


bien reconoce que la fuente principal del tomo «español-francés» del Diccionario nuevo de las lenguas española y francesa de Sobrino es el Tesoro... de César Oudin, demuestra también que el lexicógrafo español lo utiliza de manera bastante crítica:

D'une part, il supprime un assez grand nombre d'entrées (environ un quart) qui se trouvaient chez Oudin. Ces suppressions ont trait à: (1) des lexèmes qui étaient devenus des archaïsmes au début du XVIII siècle; (2) des lexèmes qui ne semblent jamais avoir été attestés chez les auteurs espagnols et pouvaient donc être considérés comme de pures inventions de Oudin; (3) les lexèmes de germanía qu'Oudin avait introduits dans son Tesoro... ${ }^{39}$

Robert A. Verdonk concluye así su demostración: «La partie E[spagnol]F[rançais] de Sobrino 1705 constitue donc une mise à jour de Oudin, une adaptation à l'évolution de l'espagnol tel qu'il s'utilisait au début du XVIII ${ }^{e}$ siècle» ${ }^{40}$. Tal adaptación era imprescindible, si nos fijamos en lo que escribe Guillaume de Maunory en su Grammaire et dictionnaire françois et espagnol [...] suivant l'usage de la Cour d'Espagne, obra publicada en 1701 con reediciones en 1704 y 1708: «[...] il n'est pas étonnant de voir un homme qui va en Espagne imbu des préceptes \& des mots de Houdin (sic), n'y être pas entendu, \& qu'il s'y trouve aussi nouveau, que s'il n'avoit jamais rien appris» ${ }^{4}$. En conclusión, podemos afirmar que Sobrino se sirvió ampliamente del Tesoro... de Oudin, erigido ya en autoridad lexicográfica, pero lo modernizó y lo adaptó a los usos lingüísticos del siglo XVIII: por consiguiente, el reproche mayor que se podría hacer a Francisco Sobrino es que no reconoció claramente su deuda con la obra del lexicógrafo francés ${ }^{42}$. No obstante, en aquel entonces se trataba de algo corriente, ya que esta práctica «se inserta[ba] en una tradición lexicográfica [...] basada en la copia o imitación como base desde la que desarrollar la propia original.... ${ }^{43}$. En efecto, como lo afirma también el profesor Messner, «es bien sabido que en la lexicografía más que en ningún otro sector de la filología los autores de obras nuevas recurren a las precedentes para copiarlas» ${ }^{44}$.

A modo de conclusión, podemos afirmar que, para elaborar su Tesoro..., César Oudin se valió de muchos materiales lexicográficos de obras anteriores erigidas ya en autoridades como los diccionarios de Nebrija, de Cristóbal de Las Casas, de Heinrich Hornkens y de Jean Palet. Pero Oudin supo completar estos préstamos con varias entradas nuevas sacadas de sus propias lecturas de obras literarias españolas o de diccionarios muy recientes como el Tesoro de la lengua castellana y española (1611) de Sebastián de Covarrubias, lo que dio al Tesoro... fama de obra innovadora. El inmenso éxito que conoció este diccionario lo convirtió, a su vez, en autoridad de

39. Verdonk, 1991, p. 2978.

40. Verdonk, 1991, pp. 2978-2979.

41. Maunory, Grammaire et dictionnaire françois et espagnol..., fol. ã III.

42. Las fuentes evocadas por Sobrino en su diccionario son las siguientes: el Dictionaire françois de P. Richelet, el Dictionnaire universel de Furetière, el Dictionnaire étymologique de G. Ménage y el Dictionarum novum latinum et gallicum de P. Dauet. En cambio, nunca cita el Tesoro... de Oudin, ni el de Vittori. Tampoco alude al Dictionnaire de l'Académie française.

43. Sáez Rivera, 2002, p. 27.

44. Messner, 2000, p. 129. 
la cual se aprovecharon muchos lexicógafos posteriores como Lorenzo Franciosini, César-Joachim Trognesius, Arnaldo De la Porte, Nicolas Mez de Braidenbach y Francisco Sobrino. Por lo tanto, también podemos afirmar con José Joaquín Martínez Egido que «un diccionario bebe de otros anteriores» ${ }^{45}$, por lo cual las obras lexicográficas de gran importancia pasan del estatus de textos innovadores al de obras de referencia, es decir, con autoridad. Este proceso es precisamente el que se produjo en el caso del Tesoro... de César Oudin cuya larga historia lo sitúa entre autoridad e innovación, como se puede comprobar en el stemma que viene a continuación.

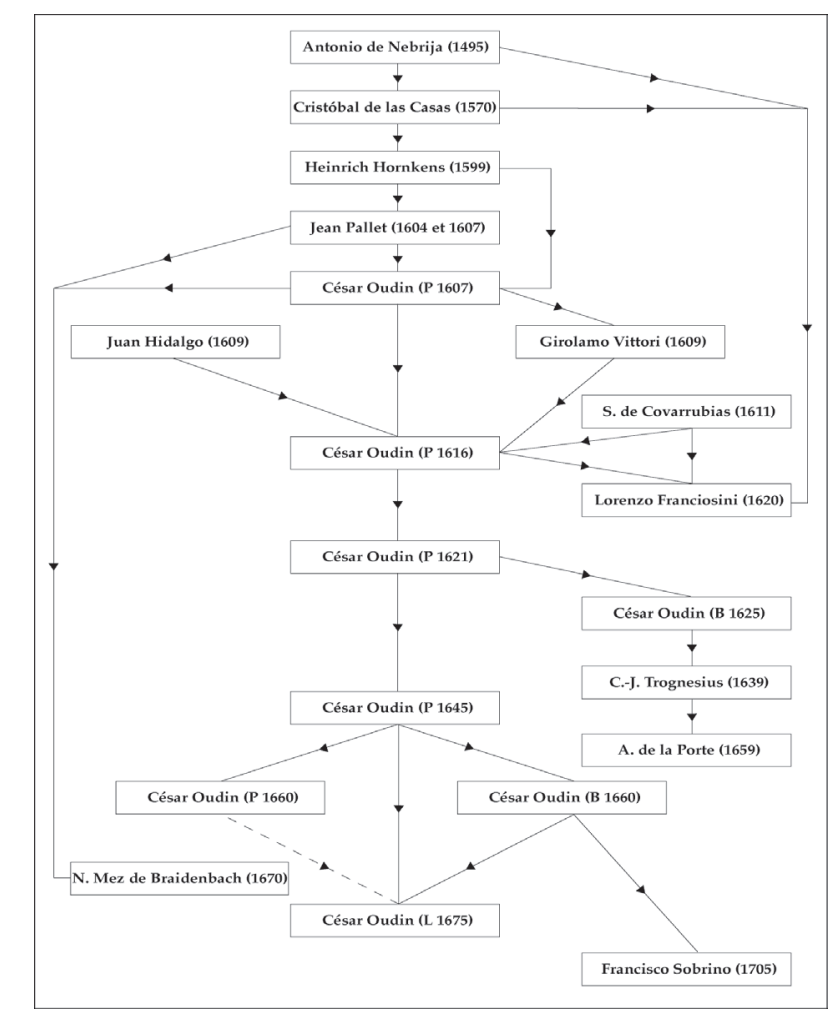

\section{BIBLIOGRAFÍA}

Cooper, Louis, «Girolamo Vittori y César Oudin: un caso de plagio mutuo», Nueva Revista de Filología Hispánica, 14, 1960a, pp. 3-20.

Cooper, Louis, «Sebastián de Covarrubias: una de las fuentes principales del Tesoro de las dos lenguas española y francesa (1616) de César Oudin», Bulletin Hispanique, 62/4, 1960b, pp. 366-397. 
Cooper, Louis, «El Recueil de Hornkens y los diccionarios de Palet y de Oudin», Nueva Revista de Filología Hispánica, 16I, 1962a, pp. 297-328.

Cooper, Louis, «Plagiarism in Spanish dictionaries of the $16^{\text {th }}$ and $17^{\text {th }}$ centuries», Hispania, 45/4, 1962b, pp. 717-720.

Gallina, Annamaria, Contributi alla storia della lessicografia italo-spagnola dei secoli XVI e XVII, collezione «Biblioteca dell' "Archivum Romanicum"», Serie I, vol. 58, Firenze, Olschki, 1959.

Gili Gaya, Samuel, Tesoro lexicográfico (1492-1726), t. I, Madrid, CSIC, 1947.

Hausmann, Franz Josef, «Der Diccionario muy copioso des Nicolas Mez (1670) ein frühes spanish-deutsches Wörterbuch», Navicula Tubingensis, Studia in honorem Antonii Tovar, ed. Francisco J. Oroz Arizcuren, Tübingen, Narr, 1984, pp. 167-171.

Martínez Egido, José Joaquín, «La utilización de fuentes en la realización de diccionarios: ¿Covarrubias (1611), fuente directa en la microestructura de Franciosini (1620)?», Estudios de lingüística Universidad de Alicante, 17, 2003, pp. 413426.

Maunory, Guillaume (de), Grammaire et dictionnaire françois et espagnol [...] suivant I'usage de la Cour d'Espagne, Paris, Veuve Claude Barbin, 1701.

Messner, Dieter, «El Diccionario de la lengua castellana de 1780: una fuente del Diccionario da lingoa portuguesa de 1793», Revista de Filología Española, 80, 2000, pp. 129-139.

Messner, Dieter, «El primer diccionario bilingüe español-alemán», s.f., disponible en: $<$ http://www.uni-salzburg.at/index.php?id=30576\&L=0> [20/01/2015].

Oudin, César, Thrésor des deux langues françoise et espagnolle. Thrésor des deux langues françoise et espagnolle..., Paris, Vve Marc Orry, 1616.

Oudin, César, Tesoro de las dos lenguas francesa y española. Thrésor des deux langues françoise et espagnole, León de Francia, J.-B. Bourlier \& L. Aubin (o Miguel Mayer o A. Beaujollin), 1675.

Pablo Núñez, Luis, El arte de las palabras. Diccionarios e imprenta en el Siglo de Oro, Mérida, Editora Regional de Extremadura, 2010, 2 vols.

Sáez Rivera, Daniel M., «Vida y obra de Francisco Sobrino», Revista Electrónica sobre Literatura Medieval y Renacimiento (LEMIR), pp. 1-33, 2002, disponible en: <parnaseo.uv.es/Lemir/Textos/Sobrino/INTRO.pdf> [31/01/2016].

Verdonk, Robert A., «El diccionario plurilingüe llamado Anónimo de Amberes (1639), reflejo de la lexicografía española en Flandes», en Actas del Primer Congreso Internacional de Historia de la Lengua Española, ed. Manuel Ariza, Antonio Salvador y Antonio Viudas, Madrid, Arco/Libros, 1988, pp. 995-1002. 
Verdonk, Robert A., «La importancia del "Recueil" de Hornkens para la lexicografía bilingüe del Siglo de Oro», Boletín de la Real Academia Española, 70, cuaderno 259, 1990, pp. 69-109.

Verdonk, Robert A., «La lexicographie bilingue espagnol-français, français-espagnol», Wörterbücher-Dictionaries-Dictionnaires: encyclopédie internationale de lexicographie, ed. Franz Josef Hausmann et al., t. III, Berlin/New York, Walter de Gruyter, 1991, pp. 2976-2987.

Verdonk, Robert A., «La dette de César Oudin envers le "Recueil" de H. Hornkens et ses conséquences pour la lexicographie espagnole du XVII siècle», Actualités de la recherche en linguistique hispanique, Actes du IVe colloque de linguistique hispanique, ed. Gilles Luquet, Limoges, PULIM, 1992a, pp. 9-23.

Verdonk, Robert A., «El "Nuevo dictionario" neerlandés-español/español-neerlandés de A. De la Porte (Amberes, 1659) y su importancia para la lexicología y la lexicografía del español de los Siglos de Oro», Actas do XIX Congreso Internacional de Lingüística e Filoloxía Románicas, ed. Ramón Lorenzo, t. II: Lexicoloxía e metalexicografía, A Coruña, Fundación «Pedro Barrié de la Maza, conde de Fenosa», 1992b, pp. 35-45.

Verdonk, Robert A., «Las ediciones bruselenses del Tesoro de C. Oudin. Estudio de sus peculiaridades léxicas», en Actes du XXe Congrès International de linguistique et de Philologie Romanes, ed. Hilty Gerold, t. IV, Tübingen, A. Francke, 1993, p. 789-800.

Zuili, Marc, César et Antoine Oudin, deux polygraphes français des XVI et XVII siècles : vie, œuvre et ébauche d'une édition critique du «Tesoro de las dos lenguas española y francesa» (1607-1675) [Habilitation à diriger des recherches], Université de Paris X-Nanterre, 2004.

Zuili, Marc, «Nuevas aportaciones sobre el hispanista francés César Oudin (1560?1625)», Thélème: Revista complutense de estudios franceses, 20, 2005, pp. 203-211, disponible en: <http://revistas.ucm.es/index.php/THEL/article/ viewFile/THEL0505110203A/33307> [20/01/2015].

Zuili, Marc, «César Oudin y la difusión del español en Francia en el siglo XVII», en La cultura del otro: español en Francia, francés en España, ed. Manuel Bruña Cuevas et al., Sevilla, Departamento de Filología Francesa de la Universidad de Sevilla, 2006, pp. 278-289, disponible en: <http://www.culturadelotro.us.es/ actasehfi/pdf/2zuili.pdf> [25/01/2015].

Zuili, Marc, «Pour une édition critique de la partie "espagnol-français" du Tesoro de las dos lenguas española y francesa de César Oudin (éd. princeps: 1607)», Quaderns de filologia. Estudis linguistics, 13, ed. Brigitte Lépinette, María José Martínez Alcalde y Emili Casanova, Valencia, Universitat de València, 2008, pp. 105-134, disponible en: <https://ojs.uv.es/index.php/qfilologia/article/ download/4029/3670> [25/01/2015]. 
Zuili, Marc, «L'enseignement du castillan en France au début du XVIIe siècle», en Les langues étrangères en Europe. Apprentissages et pratiques (1450-1720), ed. Marc Zuili y Susan Baddeley, Paris, Presses de l'université de Paris Sorbonne (PUPS), 2012, pp. 91-108.

Zuili, Marc, «Les mystères de l'évolution du Tesoro de las dos lenguas española y francesa de César Oudin entre 1607 et 1675», HispanismeS, Hommage à Geneviève Champeau, ed. Christian Lagarde y Philippe Rabaté, 3, 2014, pp. 83100, disponible en: <http://www.hispanistes.org/images/PDF/SHF HispanismeS 3 ZUILI Marc.pdf> [15/01/2015]. 